\title{
Longitudinal Effects of Botulinum Toxin Injections on Voice-Related Quality of Life (V-RQOL) for Patients with Adductory Spasmodic Dysphonia
}

\author{
Norman D. Hogikyan, Walter P. Wodchis, Constance Spak, and Paul R. Kileny \\ Department of Otolaryngology, University of Michigan Medical Center, Ann Arbor, Michigan
}

\begin{abstract}
Summary: Adductory spasmodic dysphonia is a focal dystonia of laryngeal muscles. Patients with this disorder typically have severe vocal difficulties, with significant functional, social, and emotional consequences. There is no widely accepted cure for this condition, however, botulinum toxin injections of the thyroarytenoid muscles are considered by most voice clinicians to be the state of the art treatment. Based on extensive experience treating patients for adductory spasmodic dysphonia, we feel that traditional means of voice assessment do not adequately measure either the disease severity or the treatment outcomes. That is, listening to or acoustically analyzing limited phonatory samples does not capture the functional, social, and emotional consequences of this disorder. These consequences will be reflected in a patient's voice-related quality of life (V-RQOL). Using a validated voice outcomes instrument, the V-RQOL Measure, the purpose of this study was to quantify longitudinal changes in the V-RQOL of patients with adductory spasmodic dysphonia who are undergoing botulinum toxin injections. Twenty-seven consecutive new patients presenting with dysphonia to our institution during an 18-month period were diagnosed with adductory spasmodic dysphonia, and treated patients were evaluated prospectively using the VRQOL Measure. Results indicated that (1) V-RQOL was initially very low for these patients, (2) botulinum toxin injections improved it significantly for each injection cycle studied, and (3) the magnitude of the treatment effect appears to change across injections. Key Words: Voice-related quality of life (V-RQOL)Botulinum toxin (Botox) - Adductory spasmodic dysphonia.
\end{abstract}

\section{INTRODUCTION}

Spasmodic dysphonia (SD) is a focal dystonia of laryngeal muscles. It reflects abnormalities in central

Accepted for publication February 12, 2001

Address correspondence and reprint requests to Norman D. Hogikyan, MD, FACS, Director, Vocal Health Center, Assistant Professor, Department of Otolaryngology, University of Michigan Medical Center, 1904 Taubman Center, 1500 E. Medical Center Drive, Ann Arbor, MI 48109-0312.

e-mail: nhogikya@umich.edu motor processing for laryngeal control, but the cause is not known. The most common form of the disorder, adductory spasmodic dysphonia (AdSD), is characterized by action-induced spasms of the adducting laryngeal muscles. This typically causes severe dysphonia, with such terms as strained, strangled, tight, or pressed often used to describe the vocal quality. ${ }^{1-4}$

Various methods have been used to characterize the severity of dysphonia and the effects of treatment 
in patients with AdSD. These have included acoustic, aerodynamic, electromyographic, spectrographic, endoscopic, and perceptual analyses. ${ }^{1,5-8}$ Such methods may help to understand the physiological changes associated with this disorder, but they will not reflect the social, emotional, and functional consequences that the patients experience in their daily activities. These consequences will be reflected in a patient's voice-related quality of life (V-RQOL).

Some authors have recognized this important point, and have designed studies to measure the emotional and functional impact that AdSD has upon patients. Cannito ${ }^{9}$ used psychometric measures of depression, anxiety, and somatic complaints to study SD patients. That study found an "affective factor" present among these patients that was not present among controls, and suggested that this may support a psychogenic basis for SD. Murry et $\mathrm{al}^{10}$ and Liu et $\mathrm{al}^{11}$ used psychometric measures of depression and anxiety to study SD patients before and after treatment with botulinum toxin (Botox) injections. Their results found significant differences between these patients and controls, with improvement seen on most measures for patients treated with Botox. These results support the contemporary viewpoint that SD is not a psychogenic disorder, but rather, that the emotional symptoms result from the voice disorder itself.

Smith et al ${ }^{12}$ studied the functional impact of SD upon work-related issues and general communication factors such as telephone use. Based upon questionnaires designed for that particular study, patients with SD were significantly more likely to indicate adverse vocational effects and difficulties with general communication than patients without dysphonia. These authors felt that it is important "to report voice symptoms from the patient viewpoint."

A variety of different treatment approaches have been explored for AdSD. These have included voice therapy, therapeutic lesions of the recurrent laryngeal nerve, partial myectomy of the thyroarytenoid (TA) muscles, laryngoplasty, and chemodenervation using Botox injections. ${ }^{1,4,13-19}$ Botox injections are the most widely accepted treatment for AdSD, with treatment effect having been measured in a variety of ways as noted above.

Outcomes research is the scientific study of the outcomes of various therapies presently utilized for a particular disease, condition, or illness. ${ }^{20}$ The goals of this type of research are to document treatment effectiveness and to create treatment guidelines for appropriate medical care. ${ }^{21}$ Driven to a certain degree by third-party payers and the societal mandate for cost-efficient and effective health care, contemporary clinical research in otolaryngology and many other medical and surgical disciplines therefore include a focus upon such investigation. ${ }^{22,23}$ From this type of research, a more complete list of treatment outcome measures emerges, including mortality, morbidity, health status, quality of life (QOL), and satisfaction with care. ${ }^{24-27}$

The Voice-Related Quality of Life (V-RQOL) Measure is a validated outcomes instrument specific for voice disorders (Appendix A). ${ }^{28,29}$ In a diverse population of voice disorders it has been shown to be valid, reliable, responsive to change, and of low burden. Ongoing investigation with this outcomes tool is designed to better understand the functional, social, and emotional consequences of dysphonia, and to help build a core of evidence-based outcomes literature for the treatment of voice disorders. The goals of the current study are therefore to (1) determine the V-RQOL of patients with untreated AdSD, (2) prospectively measure the treatment effect of Botox injections upon this value, (3) determine if the measured treatment effect changes with subsequent injections over time, and (4) enhance understanding of the utility of the V-RQOL measure.

\section{MATERIALS AND METHODS}

\section{Patient population and data acquisition}

Twenty-seven new patients presenting with dysphonia to the University of Michigan vocal health program over a recent 18 -month period were diagnosed with AdSD. Diagnoses were made by experienced voice clinicians. As there is no single test for determining the presence of spasmodic dysphonia, diagnoses were made based upon a combination of history, vocal capabilities, and laryngeal videostroboscopy. Typical historical features included gradual onset of the condition, and fluctuation in symptoms but no periods of completely normal voice since disease onset. Aggravation of symptoms with stressful circumstances was nearly universal. Perceptual diagnostic features of the voice included a pressed or 
strained vocal quality with intermittent stoppages, and stoppages were typically more pronounced with vowels. Often the singing voice was clearer than the speaking voice, and the upper voice clearer than the lower tones. Each of these patients completed the VRQOL Measure, and an overall self-rating of voice quality. Twenty-one of these patients underwent at least one round of bilateral TA muscle Botox injections with follow-up during the study period. Three of the original patients chose not to undergo injections, while three others underwent their first injection after the period of this study was completed.

The technique of injection has been previously described by other authors, ${ }^{1}$ and all were performed by the same fellowship trained laryngologist (NDH). Highlights included an EMG guided percutaneous approach via the cricothyroid membrane following local anesthesia of the skin and topical anesthesia of the airway. All patients were treated with bilateral TA muscle injections of Botox Type A (Allergan, Inc., Irvine, CA), and the usual starting dosage at our institution is 1.0 unit per vocal fold. Dosage was decreased or increased in small increments, if necessary, with subsequent injections to decrease side effects or to enhance duration of benefit.

Patients returned for assessment and completed the V-RQOL Measure and overall voice rating six to eight weeks following the initial injection. Any patient felt to have persistent breathy dysphonia as a side effect of the injection at that point was scheduled for a repeat six-week follow-up assessment in the clinic. Otherwise, patients were instructed to call for a repeat injection when they felt that their vocal symptoms had returned.

This cycle of preinjection and postinjection VRQOL assessments was repeated prospectively for each subsequent injection. Follow-up questionnaires were mailed to patients for later injections and returned by self-addressed envelope unless the patient had prolonged side effects with the initial injection. Any such patient was seen in the clinic for follow-up assessments. Fourteen patients underwent at least two injections, three patients at least three injections, and one patient had four injections with follow-up during the study period. Treated patients also prospectively completed a preinjection and postinjection self-assessed overall voice quality categorical rating of poor, fair, good, very good, or excellent.

\section{Statistical analyses}

Survey responses were recorded from the pretreatment and posttreatment questionnaires. Treatment dates and demographic data were recorded from clinical records. SAS v6.12 and Microsoft Excel were used to manage the data and calculate results, including statistical tests.

Summary demographic characteristics were calculated for all patients diagnosed with AdSD $(N=27)$. V-RQOL summary scores as well as social-emotional (SE) and physical functioning (PF) domain scores were calculated based on the respective scoring algorithms (Appendix B). ${ }^{28}$ These scores were based on 10,4 , and 6 items, respectively. The formula used to calculate all three scores provides an index of $\mathrm{V}$ RQOL on a scale of 0 to 100 , where 0 is very poor VRQOL and 100 is the highest possible V-RQOL that one could expect to attain.

Mean V-RQOL, SE, and PF domain scores and standard deviations were calculated for all new patients diagnosed with AdSD ( $N=27)$. Pretreatment and posttreatment scores, and longitudinal change scores were calculated for each treated patient $(N=21)$. Matched $t$ tests were used to test whether these changes were significant. $p$ values reported in the accompanying tables were based on two-tailed significance tests. Self-assessed voice quality ratings and changes in rating category with treatment were also calculated.

Analyses for second treatments were performed for patients who completed a second treatment and follow-up sequence $(N=14)$. Matched $t$ tests for pre and post differences, and differences in longitudinal change scores between the first and second treatments were performed. Time to second treatment and changes in the patient's categorical voice ratings were also calculated.

Because there was only one patient with four treatments, third and fourth treatment effects were merged in the analysis. Hence there were three patients with more than two treatments, but there were four "third/fourth" treatments. Analyses for third/fourth treatments were the same as for patients with second treatments. In addition, differences in time to treatment between successive treatments (e.g., time between second and third treatment) were tested for those patients with more than two treatments. 


\section{RESULTS}

The mean age for the entire study population was 51.9 years, with a range of 24.7 to 78.9 years. There were 23 women and 4 men, for a female preponderance of $85 \%$. The initial V-RQOL scores and standard deviations for this entire population are shown in Table 1. Previously published normative V-RQOL scores for a nonvoice disorder (normal) patient population were: overall score 98.0, SE domain 98.8, and PF domain 97.3.28

Preinjection and postinjection V-RQOL and voice rating data for all patients who underwent at least one injection are shown in Table 2. The same V-RQOL data are also represented graphically for visual clarity in Figure 1. Average time to follow-up was 6.5 weeks. Large improvements in overall and domain V-
RQOL mean scores were seen with treatment, and all patients had at least $\mathrm{a}+1$ change in global voice quality categorical rating. A +1 change meant, for example, improving from poor to fair or from fair to good, with greater numerical changes indicating greater degrees of categorical improvement. Changes in VRQOL overall and domain scores were statistically significant with $p<0.000$.

Preinjection and postinjection V-RQOL and voice rating data for all patients who underwent at least two injections $(N=14)$ are shown in Table 3, with the same V-RQOL data also represented graphically in Figure 2. Average time to follow-up was 6.6 weeks for the first injection, and 7.9 weeks for the second. The mean time interval between the first and second treatments was 21 weeks with a standard deviation of

TABLE 1. Mean V-RQOL and Domain Scores (SE = social-emotional, $P F=$ physical functioning) for Overall Group of New AdSD Patients, and by Gender

\begin{tabular}{|c|c|c|c|c|c|c|}
\hline & \multicolumn{2}{|c|}{ V-RQOL } & \multicolumn{2}{|c|}{ V-RQOL-SE } & \multicolumn{2}{|c|}{ V-RQOL-PF } \\
\hline & Mean & SD & Mean & SD & Mean & SD \\
\hline Overall Group (N=27) & 30.56 & 19.20 & 33.10 & 22.66 & 28.86 & 19.70 \\
\hline \multicolumn{7}{|l|}{ Gender } \\
\hline Male $(N=4)$ & 28.13 & 13.44 & 35.94 & 10.67 & 22.92 & 16.14 \\
\hline Female $(N=23)$ & 30.98 & 20.24 & 32.61 & 24.28 & 29.89 & 20.39 \\
\hline
\end{tabular}

Abbreviation: SD, standard deviation.

TABLE 2. Mean V-RQOL and domain scores ( $S E=$ social-emotional, $P F=$ physical functioning) preinjection and postinjection for first injection of all patients $(N=21)$ with at least one Botox injection (differences for preinjection versus postinjection scores significant with $\mathrm{p}<0.000$ ). Global self-rated voice quality data preinjection and change postinjection also listed ( $a+1$ change represents improvement by one category).

\begin{tabular}{|c|c|c|c|c|c|c|}
\hline & \multicolumn{2}{|c|}{ V-RQOL } & \multicolumn{2}{|c|}{ V-RQOL-SE } & \multicolumn{2}{|c|}{ V-RQOL-PF } \\
\hline & Mean & SD & Mean & SD & Mean & SD \\
\hline Pretreatment & 28.68 & 17.14 & 29.76 & 20.15 & 27.95 & 17.51 \\
\hline Posttreatment & 85.60 & 9.52 & 86.90 & 10.44 & 84.72 & 10.32 \\
\hline Change & 56.92 & 17.88 & 57.14 & 21.86 & 56.77 & 18.64 \\
\hline \multirow[t]{2}{*}{ Pretx voice rating } & Poor & Fair & Good & Very Good & Excellent & Missing \\
\hline & 14 & 6 & 0 & 0 & 0 & 1 \\
\hline \multirow[t]{2}{*}{ Change in voice rating } & 0 & +1 & +2 & +3 & +4 & Missing \\
\hline & 0 & 4 & 6 & 5 & 5 & 1 \\
\hline
\end{tabular}

Abbreviation: SD, standard deviation. 


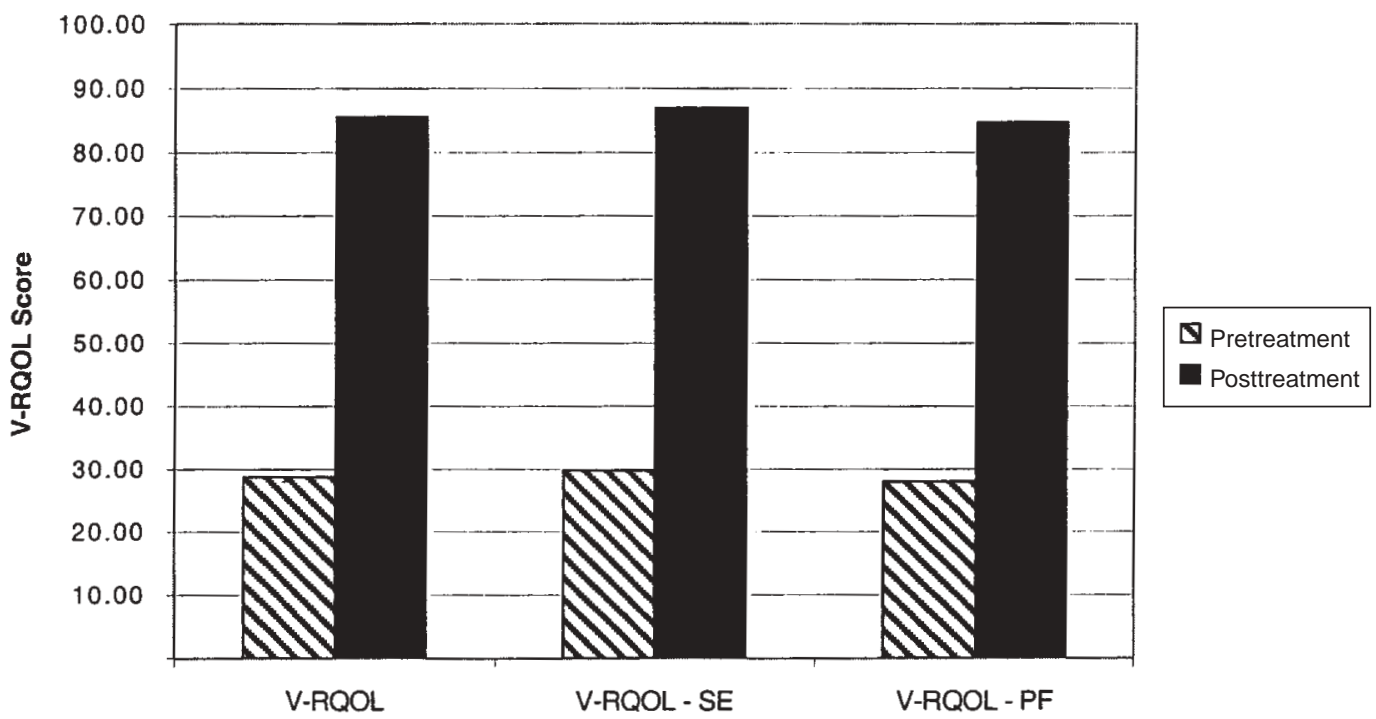

FIGURE 1. Graph of mean V-RQOL and domain scores ( $\mathrm{SE}=$ social-emotional, $\mathrm{PF}=$ physical functioning) preinjection and postinjection for first injection of all patients $(N=21)$ with at least one Botox injection (differences for preinjection versus postinjection scores significant with $p<0.000)$.

TABLE 3. Mean V-RQOL and domain scores ( $S E=$ social-emotional, $P F=$ physical functioning) preinjection and postinjection for first and second injections of all patients $(N=14)$ with at least two Botox injections (differences for preinjection versus postinjection scores significant with $p \leq 0.001$ in each case). Differences in change with treatment across first and second injections significant with $p \leq 0.002$. Global self-rated voice quality data preinjections and changes postinjections also listed $(a+1$ change represents improvement by one category).

\begin{tabular}{|c|c|c|c|c|c|c|}
\hline & \multicolumn{2}{|c|}{ V-RQOL } & \multicolumn{2}{|c|}{ V-RQOL-SE } & \multicolumn{2}{|c|}{ V-RQOL-PF } \\
\hline & Mean & SD & Mean & SD & Mean & SD \\
\hline \multicolumn{7}{|l|}{ Pretreatment } \\
\hline First treatment & 25.18 & 14.85 & 23.66 & 16.29 & 26.19 & 16.70 \\
\hline Second treatment & 42.32 & 22.99 & 42.41 & 26.08 & 42.26 & 22.58 \\
\hline \multicolumn{7}{|l|}{ Posttreatment } \\
\hline First treatment & 84.46 & 8.61 & 84.82 & 10.88 & 84.23 & 8.68 \\
\hline Second treatment & 74.29 & 22.69 & 75.00 & 24.64 & 73.81 & 22.95 \\
\hline \multicolumn{7}{|l|}{ Change } \\
\hline First treatment & 59.29 & 19.13 & 61.16 & 23.02 & 58.04 & 20.05 \\
\hline Second treatment & 31.96 & 20.01 & 32.59 & 29.23 & 31.55 & 16.63 \\
\hline Pretx voice rating & Poor & Fair & Good & Very Good & Excellent & Missing \\
\hline First treatment & 11 & 3 & 0 & 0 & 0 & 0 \\
\hline Second treatment & 5 & 9 & 0 & 0 & 0 & 0 \\
\hline Change in voice rating & 0 & +1 & +2 & +3 & +4 & Missing \\
\hline First treatment & 0 & 3 & 4 & 4 & 3 & 0 \\
\hline Second treatment & 2 & 6 & 2 & 3 & 0 & 1 \\
\hline
\end{tabular}

Abbreviation: SD, standard deviation. 


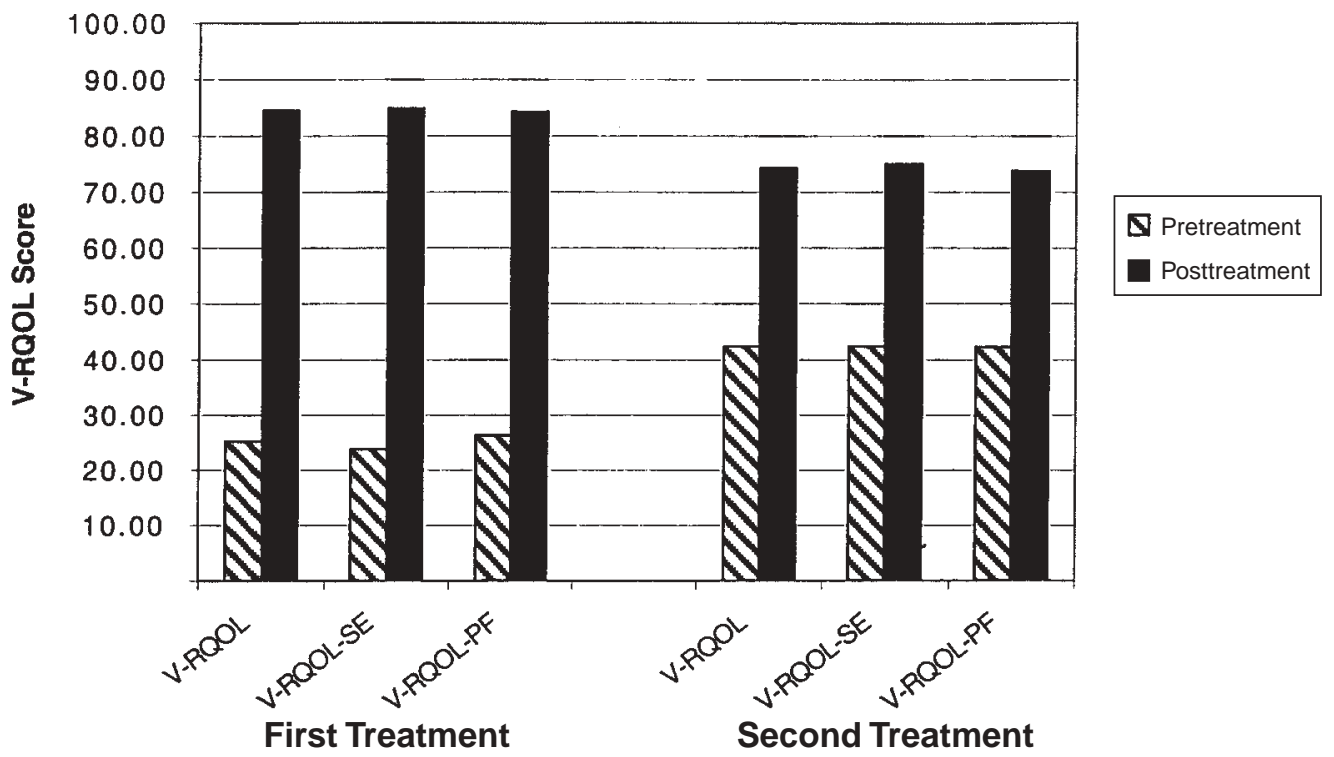

FIGURE 2. Graph of mean V-RQOL and domain scores ( $\mathrm{SE}=$ social-emotional, $\mathrm{PF}=$ physical functioning) preinjection and postinjection for first and second injections of all patients $(N=14)$ with at least two Botox injections (differences for preinjection versus postinjection scores significant with $p \leq 0.001$ in each case). Differences in change with treatment across first and second injections significant with $p \leq 0.002$.

5.8 weeks. Large improvements in overall and domain V-RQOL mean scores were seen with either the first or second treatment, and these treatment changes were statistically significant with $p \leq 0.001$ in each case. Most patients had at least a +1 change in global voice quality rating. The changes in VRQOL overall and domain scores were significantly different $(p \leq 0.002)$ across the first and second treatments, with the second treatment changes being less.

Preinjection and postinjection V-RQOL and voice rating data for all patients who underwent at least three injections are shown in Table 4, with the same V-RQOL data also represented graphically in Figure 3. For the first and second injections $N=3$, while $N=4$ for the combined third/fourth injection data. Average time to follow-up was 6.3 weeks for the first injection, and 7.3 weeks for the second or third/fourth injections. The mean time interval between injections was 19 weeks between the first and second, and 26 weeks between second and third injections or third and fourth injections. Great improvements were again seen in overall and domain V-RQOL mean scores with each treatment, and the changes with treatment were statistically significant ( $p \leq$ to 0.050 ) in each case. Most patients again had at least a +1 change in global self-rated voice quality.

\section{DISCUSSION}

It likely does not take long for any voice clinician who cares for AdSD patients to realize what a devastating disorder this can be. Effects of the condition will manifest in many aspects of these patients' lives. Clinical examination of the voice itself will not capture all the consequences of this disorder. Thus, this study was designed to measure the V-RQOL of patients with AdSD, and the treatment effects of Botox.

It is clear from this data that untreated patients with AdSD have a very low V-RQOL. This is true for the overall score as well as domain scores, with values of approximately 30 in each case. As noted earlier, previously published normals were in the upper 90 s on the scale of 0 to $100 .{ }^{28}$ The average score of 30 is also lower than the previously published means in the low 50s for a diverse group of 103 dysphonic patients who self-rated their voices as poor or fair. Thus, the AdSD patients have a low V-RQOL even when compared to a general dysphonic population seeking treatment.

The treatment effect of the first Botox injection upon V-RQOL is compelling. Improvements of close to 60 points for domain and overall V-RQOL scores represent dramatic changes. The clinical measure- 
TABLE 4. Mean V-RQOL and domain scores (SE = social-emotional, $P F=$ physical functioning) preinjection and postinjection for first, second, and third/fourth injections of all patients ( $N=3$, except $N=4$ for third/fourth injections) with at least three Botox injections (differences for preinjection versus postinjection scores significant with $p<0.050$ in each case). Differences in change with treatment across first, second, and third/fourth injections not significant with $p>0.100$ in each case. Global self-rated voice quality data preinjections and changes postinjections also listed ( $a+1$ change represents improvement by one category).

\begin{tabular}{|c|c|c|c|c|c|c|}
\hline & \multicolumn{2}{|c|}{ V-RQOL } & \multicolumn{2}{|c|}{ V-RQOL-SE } & \multicolumn{2}{|c|}{ V-RQOL-PF } \\
\hline & Mean & SD & Mean & SD & Mean & SD \\
\hline \multicolumn{7}{|l|}{ Pretreatment } \\
\hline First treatment & 24.17 & 17.74 & 16.67 & 19.09 & 29.17 & 18.16 \\
\hline Second treatment & 41.67 & 15.28 & 41.67 & 7.22 & 41.67 & 22.05 \\
\hline Third/fourth treatment & 22.92 & 10.24 & 21.88 & 11.97 & 23.61 & 10.21 \\
\hline \multicolumn{7}{|l|}{ Posttreatment } \\
\hline First treatment & 89.17 & 6.29 & 91.67 & 9.55 & 87.50 & 4.17 \\
\hline Second treatment & 94.17 & 8.04 & 100.00 & - & 90.28 & 13.39 \\
\hline Third/fourth treatment & 70.63 & 24.78 & 64.06 & 24.67 & 75.00 & 26.13 \\
\hline \multicolumn{7}{|l|}{ Change } \\
\hline First treatment & 65.00 & 17.32 & 75.00 & 16.54 & 58.33 & 18.16 \\
\hline Second treatment & 52.50 & 7.50 & 58.33 & 7.22 & 48.61 & 8.67 \\
\hline Third/fourth treatment & 47.71 & 26.06 & 42.19 & 25.71 & 51.39 & 28.40 \\
\hline Pretx voice rating & Poor & Fair & Good & Very Good & Excellent & Missing \\
\hline First treatment & 2 & 1 & 0 & 0 & 0 & 0 \\
\hline Second treatment & 0 & 3 & 0 & 0 & 0 & 0 \\
\hline Third/fourth treatment & 1 & 3 & 0 & 0 & 0 & 0 \\
\hline Change in voice rating & 0 & +1 & +2 & +3 & +4 & Missing \\
\hline First treatment & 0 & 1 & 0 & 1 & 1 & 0 \\
\hline Second treatment & 1 & 1 & 0 & 1 & 0 & 0 \\
\hline Third/fourth treatment & 1 & 2 & 0 & 1 & 0 & 0 \\
\hline
\end{tabular}

Abbreviation: SD, standard deviation.

ment of V-RQOL seems to capture well the essence of what often seems subjectively to be a life-changing treatment. Global voice self-ratings also show how the patients' perceptions of their voices improved markedly.

The data for subsequent injections also demonstrate a large and statistically significant beneficial effect of Botox on the V-RQOL of AdSD patients. This is true for the group of patients who had at least two injections, and is also true for the group who had at least three injections. Most of the patients in these groups also had at least a one category improvement in their global self-rating of voice quality after Botox treatment. Thus, it can be stated that a statistically and clinically significant effect of Botox injections as measured by V-RQOL exists for at least three injections. Experience suggests that a beneficial treatment effect can be produced for a much greater number of injection cycles, and ongoing V-RQOL research with this cohort will likely prove to be important for rigorous documentation of this clinical perception.

The two groups with multiple injections showed contrasting results with respect to differences in treatment effect between injections. Given the very small $N$ in the three or more injections group, that data must be interpreted very cautiously. Results with the two-injection group, though, merit additional discussion.

For those patients $(N=14)$ with at least two injections, the magnitude of the treatment effect with the 


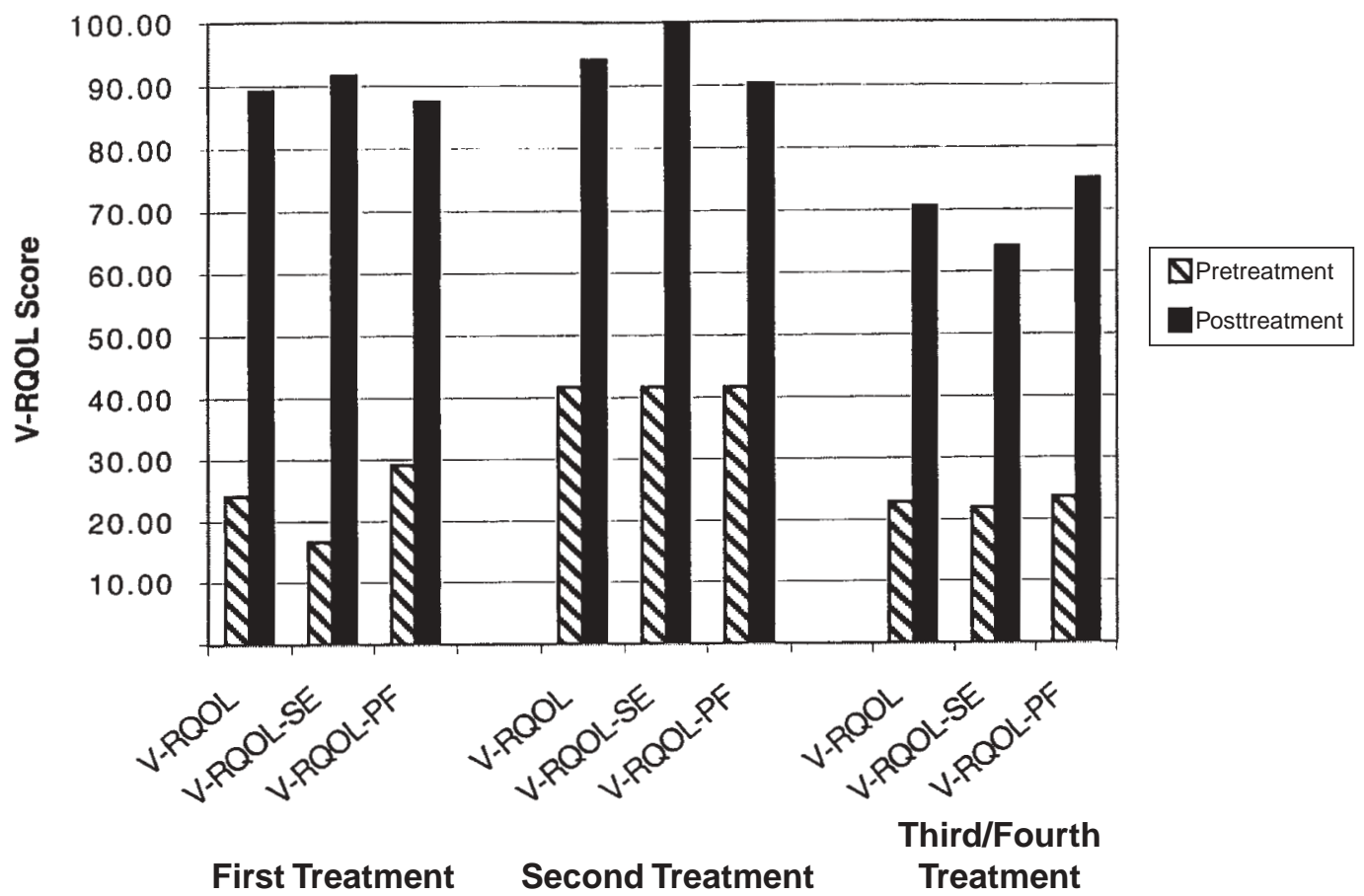

FIGURE 3. Graph of mean V-RQOL and domain scores ( $\mathrm{SE}=$ social-emotional, $\mathrm{PF}=$ physical functioning) preinjection and postinjection for first, second, and third/fourth injections of all patients ( $N=3$, except $N=4$ for third/fourth injections) with at least three Botox injections (differences for preinjection versus postinjection scores significant with $p<0.050$ in each case). Differences in change with treatment across first, second, and third/fourth injections not significant with $p>0.100$ in each case.

second injection was significantly less than that with the first injection. It is true that the mean pretreatment scores were higher before the second injections, however this alone does not account for the difference across treatments. There are two main possible interpretations for the measured difference in treatment effect. The first would be that the effect of Botox is indeed less, and that the voice quality does not reach the same levels. A second explanation would be that the patients' expectations and perceptions change once they have experienced the initial effect of a Botox treatment. That is, they begin to think of their own vocal capabilities relative to normal voices rather than to their original, severely dysphonic voice. The fact that the preinjection V-RQOL scores were higher for the second injections would support this viewpoint, as it indicates that patients return for reinjection without letting their V-RQOL return to preinjection levels. Thus, the patient-centered outcome of V-RQOL captures the evolving patient expectations and perceptions. Clinical experience with this and other disorders led us to favor the latter explanation, however, future work will likely be necessary to further define this point. Research continues with this cohort of patients and all new AdSD patients at our institution, and this larger $N$ in the multiple injection groups will be very helpful in characterizing longitudinal trends.

\section{CONCLUSIONS}

In this prospective study, patients with AdSD were shown to have a very poor V-RQOL. Botox injections of the TA muscles had a large and statistically significant beneficial effect upon this value for each injection cycle studied. The magnitude of the effect as measured by V-RQOL appears greatest with the initial injection. This further illustrates the utility of the V-RQOL Measure in the assessment of dysphonic patients. 


\section{REFERENCES}

1. Blitzer A, Brin MF, Stewart CF. Botulinum toxin management of spasmodic dysphonia (laryngeal dystonia): a 12year experience in more than 900 patients. Laryngoscope. 1998;108:1435-1441.

2. Blitzer A, Brin MF, Fahn S, Lovelace RE. Clinical and laboratory characteristics of laryngeal dystonia: a study of 110 cases. Laryngoscope. 1998;98:636-640.

3. Brin MF, Fahn S, Blitzer A, Ramig LO, Stewart C. Movement disorders of the larynx. In: Blitzer A, Brin MF, Sasaki CT, Fahn S, Harris K, eds. Neurological Disorders of the Larynx. New York, NY: Thieme; 1992:240-248.

4. Blitzer A, Brin MF. Laryngeal dystonia: a series with botulinum toxin therapy. Ann Otol Rhinol Laryngol. 1991;100: 85-90.

5. Zwirner P, Murry T, Swenson MD, Woodson GE. Effects of botulinum toxin therapy in patients with adductor spasmodic dysphonia: acoustic, aerodynamic and videoendoscopic findings. Laryngoscope. 1992;4:400-406.

6. Ludlow C. Treatment of speech and voice disorders with botulinum toxin. JAMA. 1990;264:2671-2675.

7. Woodson GE, Zwirner P, Murry T, Swenson MD. Use of flexible fiberoptic laryngoscopy to assess patients with spasmodic dysphonia. $J$ Voice. 1991;5:85-91.

8. Truong DD, Rontal M, Rolnick M, Aronson AE, Mistura K. Double-blind controlled study of botulinum toxin in adductor spasmodic dysphonia. Laryngoscope. 1991;101:630634.

9. Cannito MP. Emotional considerations in spasmodic dysphonia: psychometric quantification. J Commun Disord. 1991;24:313-329.

10. Murry T, Cannito MP, Woodson GE. Spasmodic dysphonia: emotional status and botulinum toxin treatment. Arch Otolaryngol Head Neck Surg. 1994;120:310-316.

11. Liu CY, Yu JM, Wang NM, et al. Emotional symptoms are secondary to the voice disorder in patients with spasmodic dysphonia. Gen Hosp Psychiatry. 1998;20:255-259.

12. Smith E, Taylor M, Mendoza M, et al. Spasmodic dysphonia and vocal fold paralysis: outcomes of voice problems and work-related functioning. $J$ Voice. 1998;12:223-232.

13. Cooper M. Recovery from spastic dysphonia by direct voice rehabilitation. Proceedings of 18th Congress of International Association of Logopedics and Phoniatrics. 1980;1:579584 .
14. Murry T, Woodson GE. Combined-modality treatment of adductor spasmodic dysphonia with botulinum toxin and voice therapy. $J$ Voice. 1995;9:460-465.

15. Dedo HH. Recurrent laryngeal nerve section for spastic dysphonia. Ann Otol Rhinol Laryngol. 1976;85:451-459.

16. Dedo HH, Izdebski K. Problems with surgical treatment of spastic dysphonia. Laryngoscope. 1983;93:268-271.

17. Dedo HH, Izdebski K. Intermediate results of 306 recurrent laryngeal nerve sections for spastic dysphonia. Laryngoscope. 1983;93:9-16.

18. Berke GS, Blackwell KE, Gerratt BR, et al. Selective laryngeal adductor denervation-reinnervation: a new surgical treatment for adductor spasmodic dysphonia. Ann Otol Rhinol Laryngol. 1999;108:227-231.

19. Genack SH, Woo P, Colton RH, et al. Partial thyroarytenoid myectomy: an animal study investigating a proposed new treatment for adductor spasmodic dysphonia. Otolaryngol Head Neck Surg. 1993;108:256-264.

20. Roper WL, Winkenwerder W, Hackbarth GM, Krakauer H. Effectiveness in health care: an initiative to evaluate and improve medical practice. New Engl J Med. 1988;319:11971201.

21. Relman AS. Assessment and accountability. The third revolution in medical care. New Engl J Med. 1988;319:12201222.

22. Piccirillo JF. Outcomes research and otolaryngology. Otolaryngol Head Neck Surg. 1994;111:764-769.

23. Piccirillo JF, Edwards DE, Haiduk AM, Thawley SE. Psychometric and clinimetric validity of the 31-item rhinosinusitis outcome measure. Am J Rhinol. 1995;9:297-306.

24. Feinstein AR. Hard science, soft data, and the challenges of choosing clinical variables in research. Clin Pharmacolog Ther. 1977;22:485-498.

25. O'Young J, McPeek B. Quality of life variables in surgical trials. J Chronic Dis. 1987;40:513-522.

26. Fries JF, Spitz PW. The hierarchy of patient outcome. In: Spilker B, ed. Quality of Life Assessments in Clinical Trials. New York, NY: Raven Press, Ltd.; 1990:25-35.

27. Gill TM, Feinstein AR. A critical appraisal of the quality of quality-of-life measurements. JAMA. 1994;272:619-626.

28. Hogikyan ND, Sethuraman G. Validation of an instrument to measure voice-related quality of life (V-RQOL). $J$ Voice. 1999; 13:557-569.

29. Hogikyan ND, Wodchis WP, Terrell JE, Bradford CR, Esclamado RM. Voice-related quality of life (V-RQOL) following type-I thyroplasty for unilateral vocal fold paralysis. $J$ Voice. In Press. 
APPENDIX A

VOICE-RELATED QUALITY OF LIFE (V-RQOL) MEASURE

UNIVERSITY OF MICHIGAN

NAME:

DATE:

We are trying to learn more about how a voice problem can interfere with your day to day activities. On this paper, you will find a list of possible voice-related problems. Please answer all questions based upon what your voice has been like over the past two weeks. There are no "right" or "wrong" answers.

Considering both how severe the problem is when you get it, and how frequently it happens, please rate each item below on how "bad" it is (that is, the amount of each problem that you have). Use the following scale for rating the amount of the problem:

$1=$ None, not a problem

$2=$ A small amount

3 = A moderate (medium) amount

$4=$ A lot

$5=$ Problem is as "bad as it can be"

\section{Because of my voice,}

How much of a problem is this?

1. I have trouble speaking loudly or being heard in noisy situations.

2. I run out of air and need to take frequent breaths when talking.

3. I sometimes do not know what will come out when I begin speaking.

4. I am sometimes anxious or frustrated (because of my voice).

5. I sometimes get depressed (because of my voice).

6. I have trouble using the telephone (because of my voice).

$\begin{array}{lllll}1 & 2 & 3 & 4 & 5 \\ 1 & 2 & 3 & 4 & 5 \\ 1 & 2 & 3 & 4 & 5 \\ 1 & 2 & 3 & 4 & 5 \\ 1 & 2 & 3 & 4 & 5 \\ 1 & 2 & 3 & 4 & 5 \\ 1 & 2 & 3 & 4 & 5 \\ 1 & 2 & 3 & 4 & 5 \\ 1 & 2 & 3 & 4 & 5 \\ 1 & 2 & 3 & 4 & 5\end{array}$

10. I have become less outgoing (because of my voice). 


\section{APPENDIX B}

\section{SCORING ALGORITHM FOR V-RQOL MEASURE}

V-RQOL General Scoring Algorithm

$$
100-\left[\begin{array}{c}
(\text { Raw Score }-\# \text { items in domain or total }) \\
(\text { Highest Possible Raw Score - \# items })
\end{array} \times 100\right]
$$

Social-Emotional Domain (Items 4,5,8,10)

$$
100-\left[\begin{array}{c}
(\text { Raw Score }-4) \\
(16)
\end{array} \times 100\right]
$$

Physical Functioning Domain (Items 1,2,3,6,7,9)

$$
100-\left[\begin{array}{c}
(\text { Raw Score }-6) \\
(24)
\end{array} \times 100\right]
$$

Total Score (Items 1-10)

$$
100-\left[\begin{array}{c}
(\text { Raw Score }-10) \\
(40)
\end{array} \times 100\right]
$$

Example for Total Score

If Raw Score is 30 (such as if a "medium problem" exists with all items), then:

$$
100-\left[\begin{array}{l}
(20) \\
(40)
\end{array} \times 100\right]=100-(0.5 \times 100)=100-50=50 \text { Standard Score }
$$

